\title{
OBSERVATIONS OF PLANETARY NEBULAE AT RADIO WAVELENGTHS
}

\author{
YeRVANT TERZIAN \\ (Arecibo Ionospheric Observatory*, Cornell University, Ithaca, N.Y., \\ U.S.A.)
}

\section{Introduction}

Planetary nebulae are weak radio sources. The radio emission of planetary nebulae is primarily due to free-free transitions in the ionized hydrogen cloud. Very large radio telescopes and sensitive receivers are needed to detect the weak radio emission of planetary nebulae. During the last 3 years about 80 planetary nebulae have been detected at some one radio frequency, and more than $60 \%$ of these have been detected at more than one frequency. Most of the measurements have been made to measure the flux densities of the nebulae and determine their radio spectra. A few interferometric observations have also been made to determine the radio widths of planetary nebulae. In some cases the radio observations have been used to determine the electron temperatures and emission measures of planetary nebulae, and also to derive the interstellar extinction at $\mathrm{H} \beta$.

Very recently the hydrogen $109 \alpha$ recombination line was detected in NGC 7027, thus opening a new radio way of studying the planetary nebulae.

\section{The Radio Observations of Planetary Nebulae}

One of the great difficulties of observing planetary nebulae at radio frequencies is the intrinsically weak radio energy which they emit. Planetary nebulae were first reliably detected in 1961 by Lynds (1961) who used the 85-foot Tatel radio telescope at the U.S. National Radio Astronomy Observatory (NRAO). Lynds was able to detect only the 5 strongest nebulae, IC 418, NGC 6543, NGC 6572, NGC 6853, and NGC 7293. From these observations approximate flux densities were derived for the observed nebulae at 1420 and $3000 \mathrm{MHz}$. Clearly radio telescopes with larger collecting apertures and more sensitive receivers were needed to continue the study of planetary nebulae at the radio frequencies.

In 1964, Menon and Terzian (1965) used the then newly completed 300-foot transit

* The Arecibo Ionospheric Observatory is operated by Cornell University with the support of the Advanced Research Projects Agency under a research contract with the Air Force Office of Scientific Research.

Osterbrock and O'Dell (eds.), Planetary Nebulae, 87-103. () I.A.U. 
radio telescope at NRAO, and measured the flux densities of 10 planetary nebulae at 750 and $1410 \mathrm{MHz}$. These improved measurements opened up the possibility of checking the recombination theory of emission from the detected nebulae by a comparison of the observed radio-frequency fluxes with the values predicted from the Balmer-line fluxes. It was found that the fluxes at the optically thin part of the continuum spectra of the planetary nebulae agreed reasonably well with those predicted by Osterbrock (1964) from the $\mathrm{H} \beta$ fluxes, using the recombination theory. A number of planetary nebulae were found to be optically thick at the lower frequencies. In particular the spectrum of NGC 7027 showed that it was optically thick even at $3000 \mathrm{MHz}$. NGC 6572, NGC 6543, and IC 418 were all found to have high optical depths at $750 \mathrm{MHz}$. This fact was used to compute the electron temperatures of these nebulae since at high optical depths the planetary nebulae radiate as black bodies. The results indicated electron temperatures of the order of $10-20 \times 10^{3}{ }^{\circ} \mathrm{K}$, and were in good agreement with the optical estimates. This work was followed by more than six surveys of planetary nebulae at radio frequencies ranging from 195 to $16200 \mathrm{MHz}$.

Slee and Orchiston (1965) presented a preliminary survey of planetary nebulae at radio wavelengths south of declination $+20^{\circ}$. The 210 -foot radio telescope at Parkes (CSIRO) was used at three frequencies $(620,1420$, and $2730 \mathrm{MHz})$ for these observations. About 50 planetary nebulae were detected at $2730 \mathrm{MHz}$. At the lower frequencies confusion problems were found to be serious and this made the flux measurements more difficult.

Observations at low frequencies to measure the fluxes of planetary nebulae at the optically thick part of their radio spectra were made by Terzian (1966) using the Arecibo Ionospheric Observatory's (AIO) 1000-foot spherical radio telescope. A survey of 130 planetary nebulae was made at $430 \mathrm{MHz}$ between declinations $-2^{\circ}$ and $+38^{\circ}$. A few observations were also made at 611 and $195 \mathrm{MHz}$. Figure 1 shows a sample observation at $430 \mathrm{MHz}$ of NGC 6720 (the Ring Nebula) made at AIO.

Twenty-six percent of the observed planetary nebulae gave measurable radio signals at $430 \mathrm{MHz}$. The lower flux-density limit for a detectable source was about $0 \cdot 1$ flux unit $\left(1 \mathrm{f} . \mathrm{u} .=10^{-26} \mathrm{Wm}^{-2} \mathrm{~Hz}^{-1}\right)$. Confusion problems at these low frequencies were very serious; at least $23 \%$ of the observed nebulae were confused with nearby radio sources. This study showed that for the thermal planetary nebulae with well determined optically thin radio spectra, their $\mathrm{H} \beta$ emission could be predicted. These $\mathrm{H} \beta$ fluxes were compared with the $\mathrm{H} \beta$ flux measurements uncorrected for interstellar extinction, and the differences gave the extinction in the direction of individual planetary nebulae. The results were compared with the extinction estimated optically, and a good agreement was found.

Recently Thompson et al. (1967) and Thompson and Colvin (1967) have measured the flux densities of more than 50 planetary nebulae at $3000 \mathrm{MHz}$, and 22 nebulae at $1420 \mathrm{MHz}$. The Caltech (CIT) two 90-foot diameter antennas were used as an interferometer, and both flux densities and angular widths of the observed planetaries 


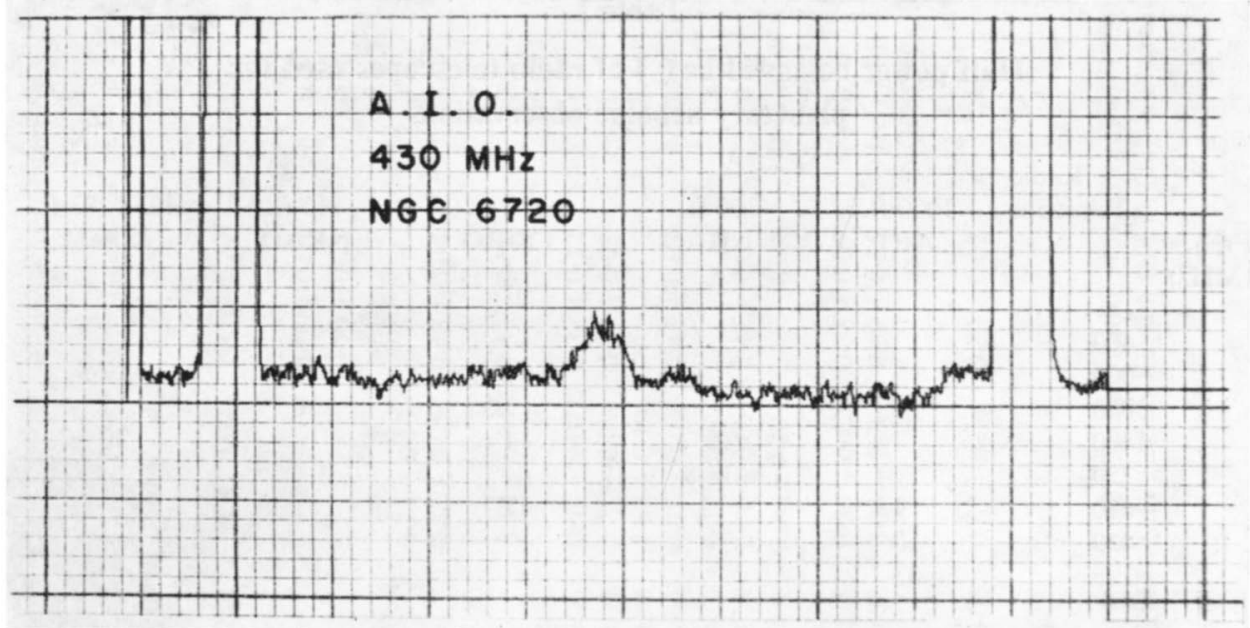

Fig. 1. Drift curve of NGC 6720 at $430 \mathrm{MHz}$ observed with the AIO 1000-foot radio telescope.

were derived. In general the radio and optical widths were found to be in good agreement.

A radio survey of planetary nebulae was also made with the Jodrell Bank Mark-II Radio Telescope by Davies et al. (1967) at 1420, 2695, and $4995 \mathrm{MHz}$. Their results are in agreement with the previous surveys, indicating that most planetary nebulae are thermal sources. NGC 7008 and NGC 7635 were reported to have non-thermal spectra, characteristic of supernova remnants and extragalactic sources.

It should also be mentioned that two high-frequency radio surveys of planetary nebulae are near completion. One survey, performed by Kaftan-Kassim at NRAO, made use of the 300 -foot transit radio telescope at $1400 \mathrm{MHz}$ and the 140 -foot radio telescope at $5000 \mathrm{MHz}$. The second survey, performed by Ehman at the University of Michigan, made use of an 85-foot radio telescope at 8000 and $16200 \mathrm{MHz}$. A few results from these surveys have been communicated to the author and are reported in the following section of this paper.

About $75 \%$ of all published flux-density measurements are in good agreement. The major uncertainties result from confusion of the planetary nebulae with non-thermal background sources. The larger the HPBW (half power beamwidth) of a radio telescope, the more serious the confusion becomes. There are about 2.5 sources per square degree with a flux density at $1400 \mathrm{MHz}$ of $\geqslant 0.1 \mathrm{f}$.u. The probability of a source being confused when observed with a 300-foot radio telescope having a HPBW of $10^{\prime}$ at $1400 \mathrm{MHz}$ is about 7\%. With the exception of the 300 -foot radio telescope at NRAO, all other existing radio telescopes have HPBW's $>10^{\prime}$ at $1400 \mathrm{MHz}$. Table 1 shows the HPBW's of the major radio telescopes used for observations of planetary nebulae. 


\section{Table 1}

\section{Half power beamwidths (') of radio telescopes used for} planetary nebulae observations

\begin{tabular}{|c|c|c|c|c|c|}
\hline $\begin{array}{r}\text { Radio } \\
\text { Telescope }\end{array}$ & $\begin{array}{l}\text { 1000-ft. } \\
\text { AIO }\end{array}$ & $\begin{array}{l}\text { 300-ft. } \\
\text { NRAO }\end{array}$ & $\begin{array}{l}210-\mathrm{ft} . \\
\text { CSIRO }\end{array}$ & $\begin{array}{l}\text { 140-ft. } \\
\text { NRAO }\end{array}$ & $\begin{array}{r}125 \times 83 \text {-ft. } \\
\text { J. Bank }\end{array}$ \\
\hline 15400 & & & & 2 & \\
\hline 5000 & & & & 6 & 7.5 \\
\hline 3000 & & & 7.5 & 10 & 15 \\
\hline 1400 & & 10 & 14 & & 30 \\
\hline 750 & & 18.8 & & & \\
\hline 610 & 9 & & 42 & & \\
\hline 430 & 10 & & & & \\
\hline 195 & 33 & & & & \\
\hline
\end{tabular}

With the exception of NGC 7293 (and possibly NGC 6853), all other planetary nebulae are point sources to the existing radio telescopes. NGC 7293 has a diameter of $12^{\prime}$ and appears as an extended source with HPBW's of $15^{\prime}$ or smaller.

\section{Radio Spectra of Planetary Nebulae}

The observations reported in the previous section clearly show that planetary nebulae have thermal radio spectra. Unlike Hil regions, planetary nebulae become opti-

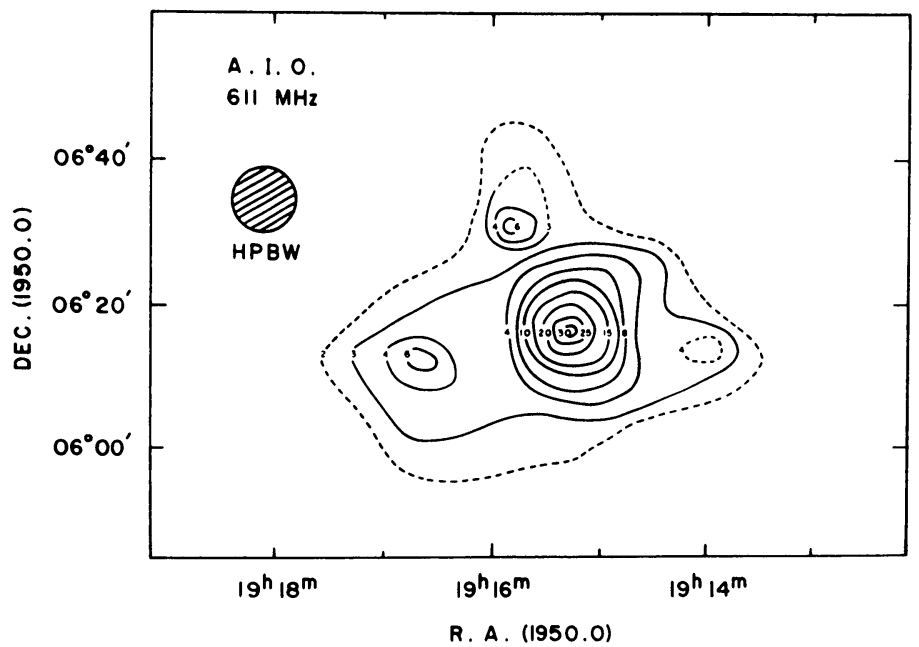

FIG. 2. Brightness temperature distribution at $611 \mathrm{MHz}$ of the region of NGC 6781. The planetary nebula coincides with the source $19^{h} 15^{m} 49^{s}$ and $06^{\circ} 29 ! 5$. 


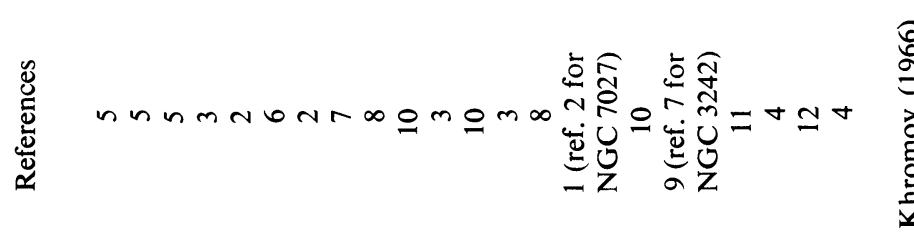

तิ

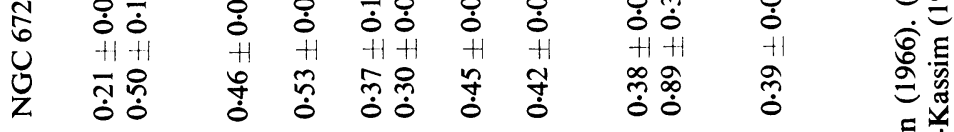

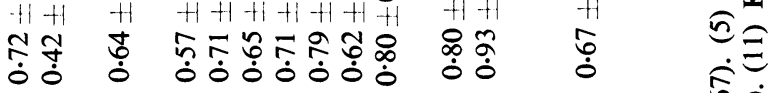

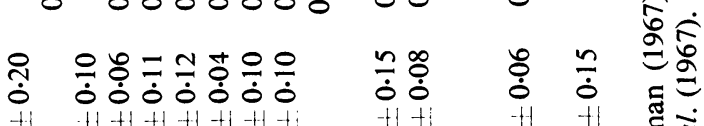
过

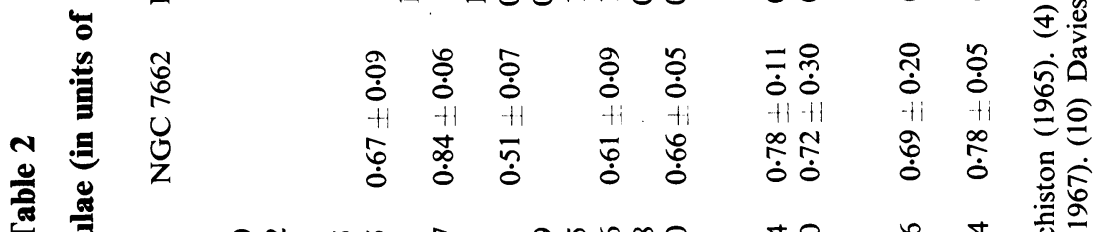

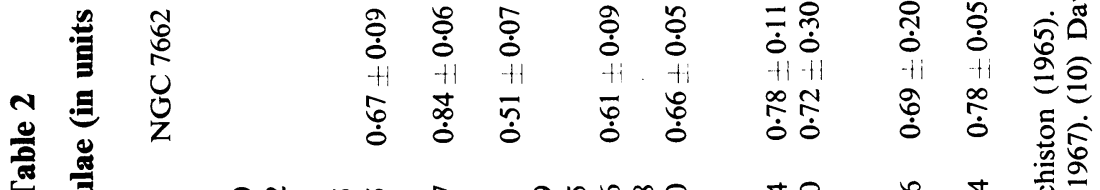

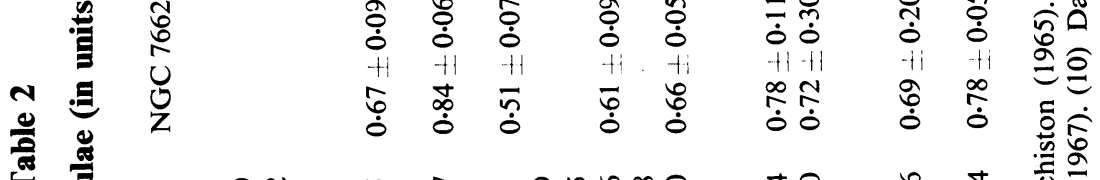
ज

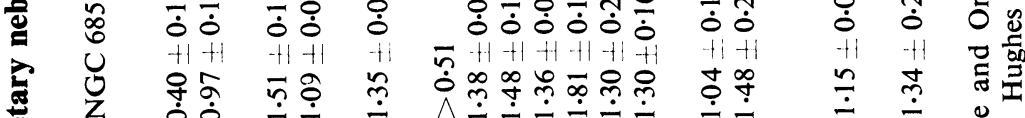

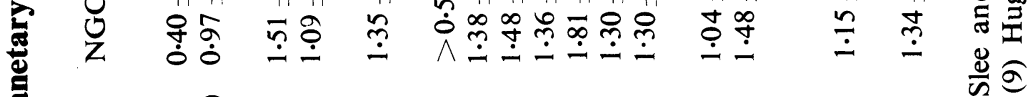

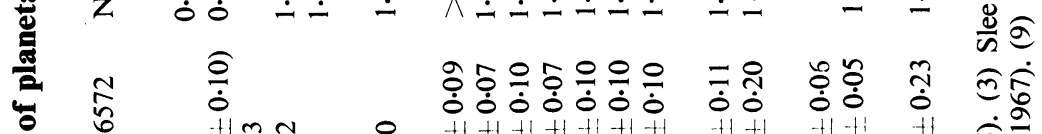

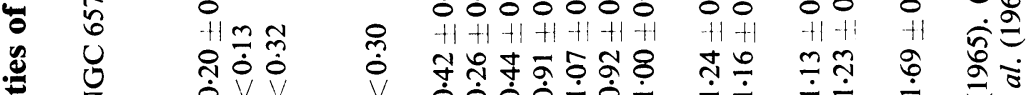

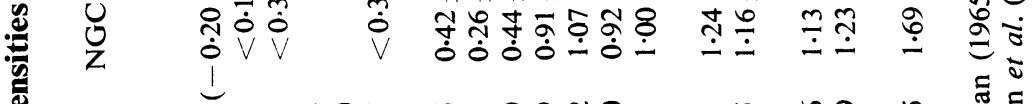

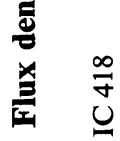

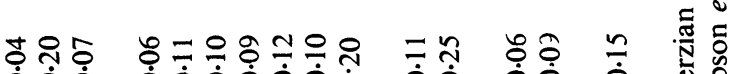

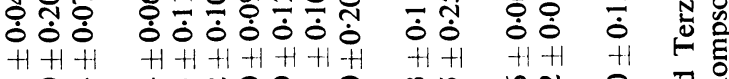

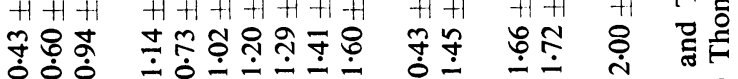
స

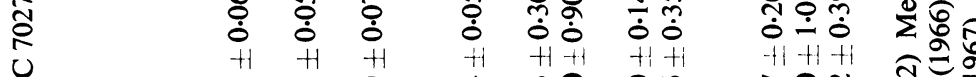

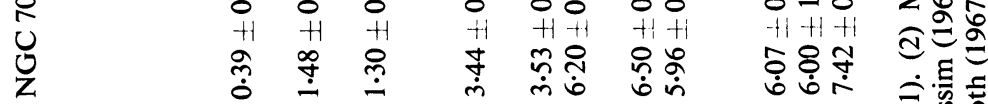

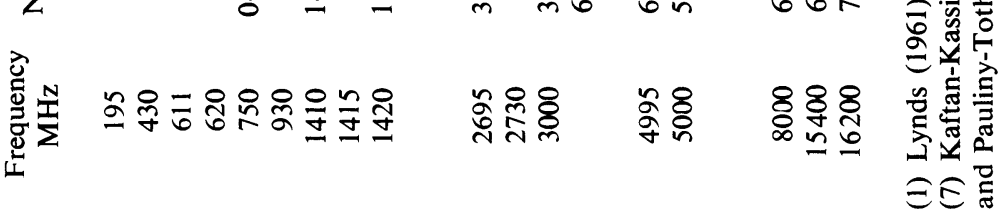


cally thick at relatively high frequencies. In a few cases radio observations have indicated possible non-thermal spectra for some planetary nebulae.

Kaftan-Kassim (1966) has examined the possible non-thermal planetary nebula NGC 3242 and has found that the excess radiation at the longer wavelengths is due to a faint arc of nebulosity about $10^{\prime} \mathrm{SW}$ of NGC 3242. The observations of NGC 6781 also indicated a steep non-thermal spectrum. Terzian (1967) surveyed the region of NGC 6781 and showed that the previous observations were seriously confused with a strong and complex background. Figure 2 shows the brightness temperature distribution at $611 \mathrm{MHz}$ of the region of NGC 6781. Both NGC 3242 and NGC 6781 now have well-established thermal spectra. Several other nebulae, like NGC 40, NGC 7008 and NGC 7635, seem to have non-thermal spectra, and more careful examinations of such cases must be made to rule out the confusion problem, if at all possible.

Table 2 summarizes the flux-density measurements of 8 planetary nebulae observed between 195 and $16200 \mathrm{MHz}$. The spectra of these nebulae are shown in Figures 3-6. It can be seen that most of the observations are in good agreement. The low flux

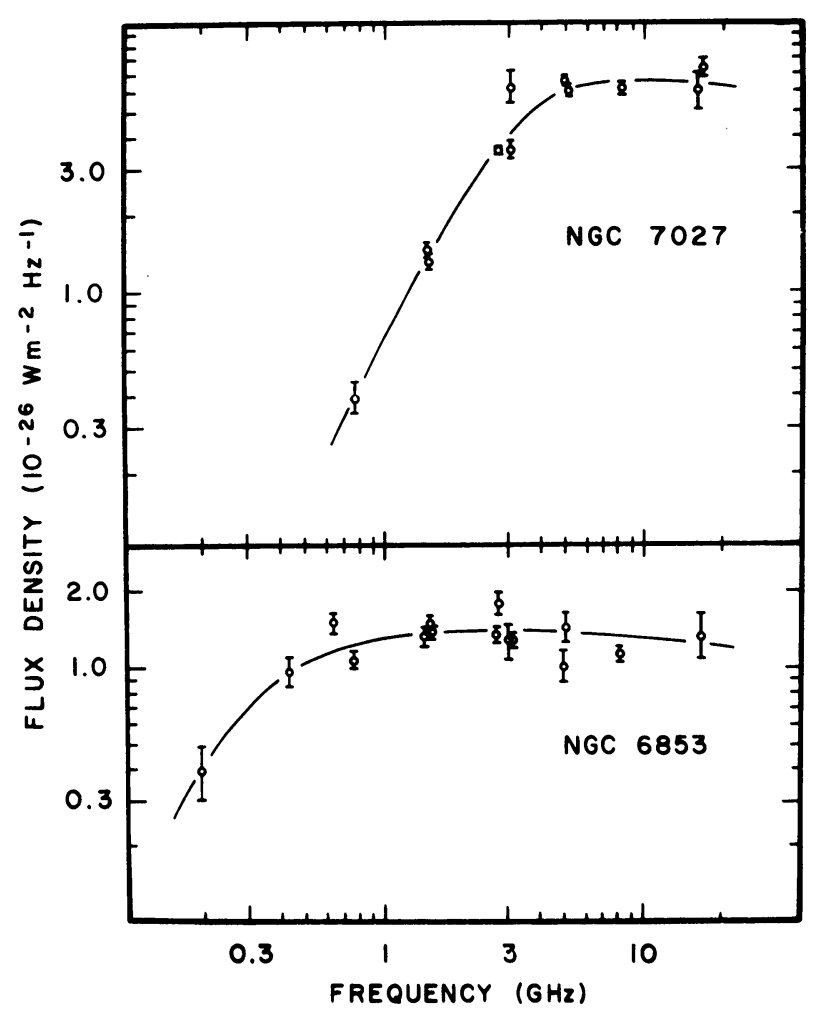

FIG. 3. Radio spectra of NGC 7027 and NGC 6853. 
density of IC 418 at $4995 \mathrm{MHz}$ reported by Davies et al. (1967) is due to positional errors during the observations (Davies, private communication).

The frequency at which the spectra of thermal sources turn to lower flux densities is a function of the emission measure. The higher the emission measure the higher is the turn-over frequency. The emission measure can easily be computed from the

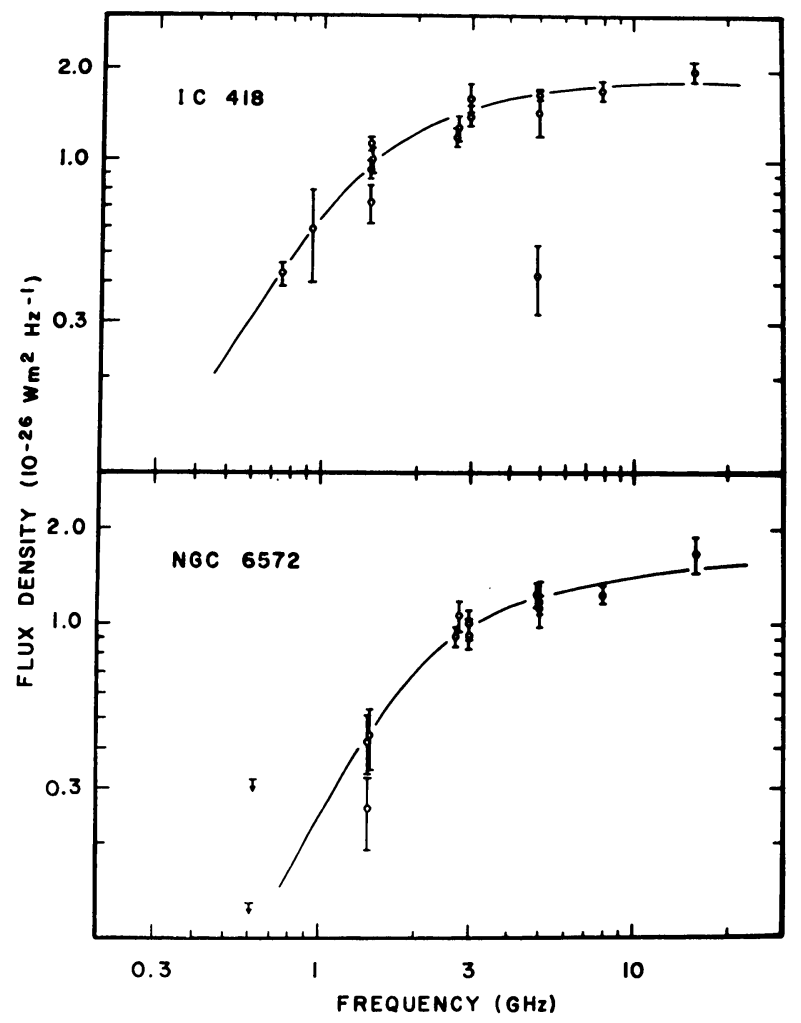

Fig. 4. Radio spectra of IC 418 and NGC 6572.

observed radio fluxes in the optically thin part of the spectra of planetary nebulae.

The absorption coefficient for free-free transitions as given by Oster (1961) can be written

$$
\kappa_{v}=\frac{N_{\mathrm{e}} N_{\mathrm{i}}}{v^{2}}\left[\begin{array}{c}
4 Z^{2} e^{6} \\
3(2 \pi)^{1 / 2} m^{3} c
\end{array}\right]\left[\frac{m}{k T_{\mathrm{e}}}\right]^{3 / 2} 2 \ln \left[\left(\frac{2 k T_{\mathrm{e}}}{\gamma m}\right)^{3 / 2}\left(\frac{m}{\pi \gamma Z e^{2} v}\right)\right],
$$

where $N_{\mathrm{e}}$ and $N_{\mathrm{i}}$ are the electron and ion densities, $Z$ is the atomic number, $e$ and $m$ are the electron charge and mass, $c$ is the velocity of light, $k$ is Boltzmann's constant, $T_{\mathrm{e}}$ is the electron temperature, $v$ is the frequency and $\gamma$ is the base of the Napierian 
logarithms to the power of Euler's constant $(\gamma \simeq 1 \cdot 78)$. Substituting numerical values for the constants in the above expression and assuming $N_{\mathrm{e}}=N_{\mathrm{i}}$, and expressing $v$ in $\mathrm{MHz}$ we have

$$
\kappa_{v}=9 \cdot 776 \times \frac{10^{-15} N_{\mathrm{e}}^{2}}{v^{2} T_{\mathrm{e}}^{3 / 2}} \ln \left(49 \cdot 503 \begin{array}{c}
T_{\mathrm{e}}^{3 / 2} \\
v
\end{array}\right) .
$$

We can also write

$$
\kappa_{v}=\zeta \begin{gathered}
N_{\mathrm{e}}^{2} \\
v^{2} T_{\mathrm{e}}^{3 / 2}
\end{gathered}
$$

where $\zeta$ varies slowly with $T_{\mathrm{e}}$ and $v$.

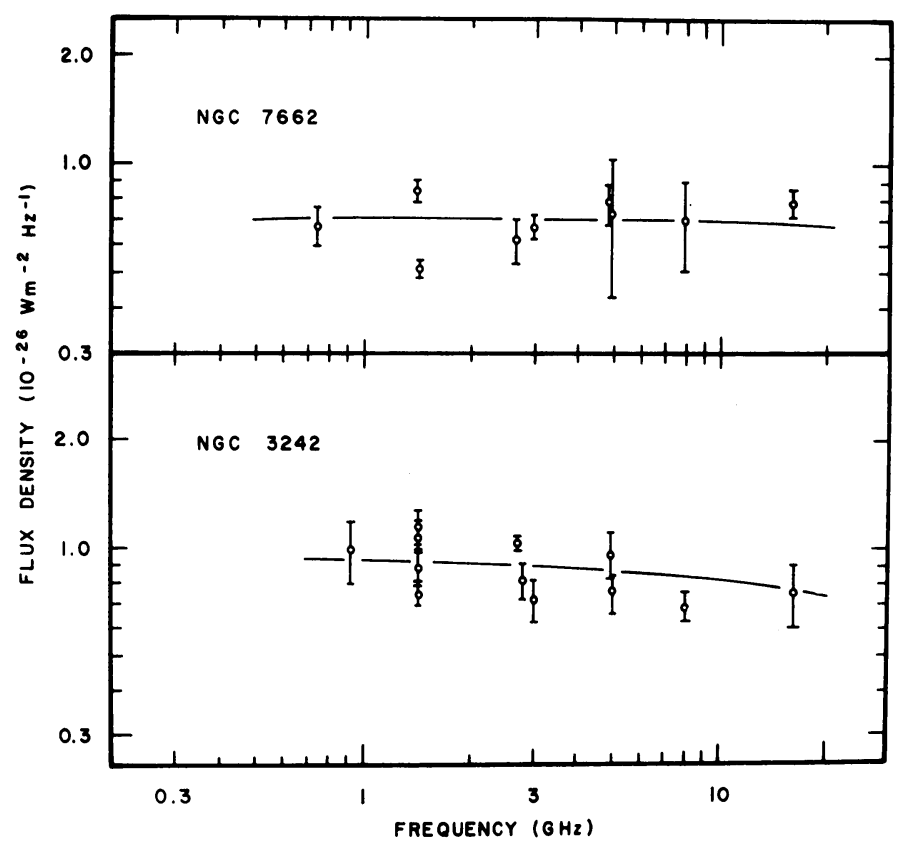

FIG. 5. Radio spectra of NGC 7662 and NGC 3242.

The emission measure $E$, defined as

$$
E=\int_{0}^{\mathrm{s}} N_{\mathrm{e}}^{2} \mathrm{~d} s,
$$

can be derived from the radio-flux measurements of planetary nebulae and can give us some estimates of the mean densities in planetary nebulae. The flux density of a planetary nebula with an apparent size at least twice as small as the HPBW of a radio 
telescope is given by

$$
S_{v}=\frac{2 k v^{2}}{c^{2}} T_{b} \Omega
$$

where $T_{\mathrm{b}}$ is the brightness temperature and $\Omega$ is the apparent solid angle of the source. Assuming that the flux of a planetary nebula is being measured at a high enough frequency so that the nebula is optically thin, we can use the approximation $T_{\mathrm{b}} \approx T_{\mathrm{e}} \tau_{v}$, where $\tau_{v}$ is the optical depth. The optical depth is defined as the integral over the absorption coefficient, and can be expressed as a function of the emission measure, i.e.

$$
\tau_{v}=\frac{\zeta E}{v^{2} T_{\mathrm{e}}^{3 / 2} .}
$$

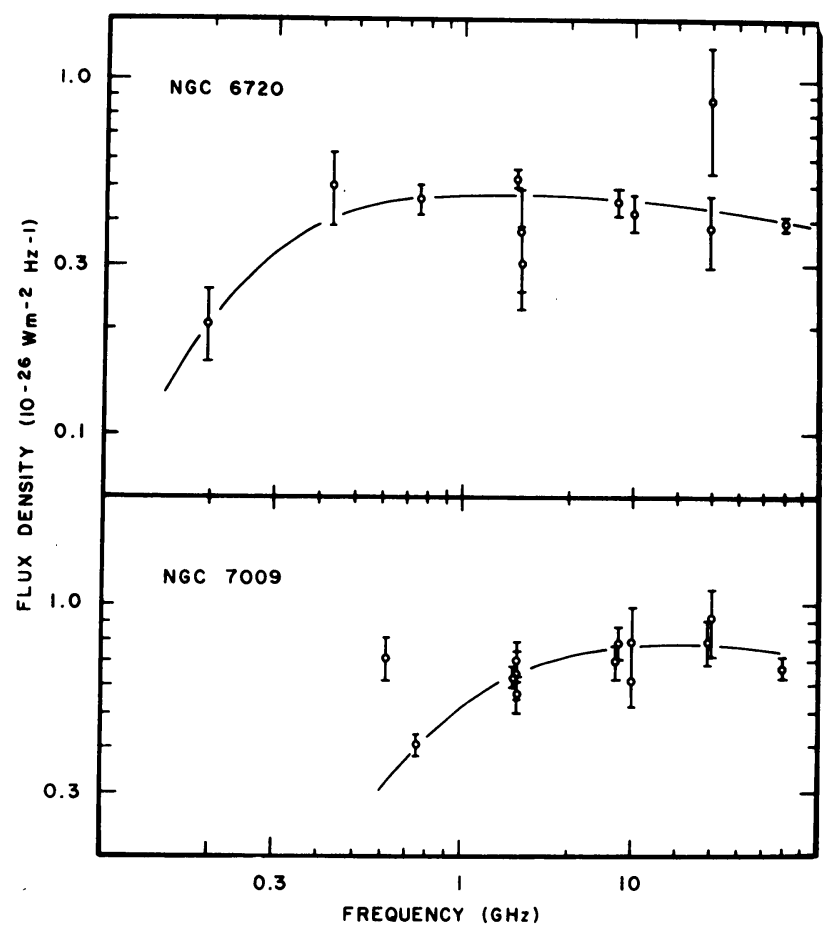

FIG. 6. Radio spectra of NGC 6720 and NGC 7009.

Thus the flux density expression (Equation 5) can be written as a function of the emission measure, or the emission measure can be expressed as,

$$
E=\frac{S_{v} T_{\mathrm{e}}^{1 / 2}}{\zeta \Omega} \cdot \frac{c^{2}}{2 k} .
$$




\section{Table 3}

\section{$H \beta$ and radio fluxes of planetary nebulae}

$\begin{array}{cccc}\text { Pl. Neb. } & \text { Corrected H } \beta \text { Flux } & \text { Reference } & \begin{array}{c}\text { Radio Flux Density } \\ \left(10^{-26} \mathrm{Wm}^{-2} \mathrm{~Hz}^{-1}\right)\end{array} \\ \left(10^{-12} \mathrm{erg} \mathrm{cm}^{-2} \mathrm{sec}^{-1}\right) & & \text { Predicted Observed Reference }\end{array}$

\begin{tabular}{|c|c|c|c|c|c|c|}
\hline NGC & 7027 & 1660 & 3 & $5 \cdot 48$ & $7 \cdot 42$ & 10 \\
\hline IC & 418 & 871 & 3 & $2 \cdot 88$ & $(2 \cdot 00)$ & 10 \\
\hline NGC & 6572 & 603 & 3 & 1.99 & (1.69) & 10 \\
\hline NGC & 6853 & 513 & 3 & 1.69 & 1.48 & 6 \\
\hline NGC & 6543 & 477 & 3 & 1.57 & 0.90 & 9 \\
\hline NGC & 7293 & 468 & 3 & 1.54 & 1.60 & 1 \\
\hline HD & 38403 & 398 & 5 & $1 \cdot 31$ & - & - \\
\hline NGC & 7662 & 275 & 3 & 0.91 & 0.78 & 8 \\
\hline NGC & 3242 & 257 & 3 & $0 \cdot 85$ & 0.97 & 8 \\
\hline NGC & 246 & 251 & 5 & $0 \cdot 83$ & $0 \cdot 24$ & 1 \\
\hline NGC & 7009 & 219 & 3 & 0.72 & 0.79 & 1 \\
\hline NGC & 6826 & 209 & 3 & 0.69 & 0.42 & 4 \\
\hline NGC & 6210 & 174 & 3 & 0.57 & $0 \cdot 46$ & 10 \\
\hline NGC & 6804 & 159 & 5 & 0.53 & $0.23 ?$ & 8 \\
\hline NGC & 6818 & 126 & 5 & 0.42 & 0.46 & 8 \\
\hline IC & 2149 & 126 & 5 & 0.42 & 0.50 & 2 \\
\hline NGC & 6720 & 123 & 3 & 0.41 & 0.42 & 4 \\
\hline IC & 4634 & 98 & 1 & 0.32 & 0.15 & 4 \\
\hline NGC & 7026 & 85 & 1 & 0.28 & $0 \cdot 27$ & 5 \\
\hline NGC & 6439 & 79 & 5 & $0 \cdot 26$ & $(-0.04)$ & 8 \\
\hline NGC & 6881 & 79 & 5 & $0 \cdot 26$ & 0.31 ? & 8 \\
\hline NGC & 6905 & 79 & 5 & $0 \cdot 26$ & $(0 \cdot 32)$ & 10 \\
\hline NGC & 40 & 79 & 5 & 0.26 & $0.34 ?$ & 5 \\
\hline NGC & 6781 & 74 & 5 & 0.24 & 0.30 & 7 \\
\hline NGC & $650-1$ & 63 & 5 & 0.21 & 0.16 & 4 \\
\hline NGC & 1535 & 63 & 5 & 0.21 & 0.21 & 1 \\
\hline NGC & 2392 & 63 & 5 & 0.21 & $0 \cdot 23$ & 4 \\
\hline NGC & 6803 & 59 & 1 & $0 \cdot 20$ & 0.11 & 8 \\
\hline IC & 2165 & 56 & 1 & $0 \cdot 19$ & $0 \cdot 22$ & 5 \\
\hline NGC & 3587 & 52 & 3 & $0 \cdot 17$ & $0 \cdot 10$ & 4 \\
\hline NGC & 4361 & 50 & 5 & $0 \cdot 17$ & $0 \cdot 20$ & 1 \\
\hline NGC & 6778 & 50 & 5 & $0 \cdot 17$ & $0 \cdot 10$ & 1 \\
\hline NGC & 6741 & 46 & 1 & $0 \cdot 15$ & 0.24 & 1 \\
\hline IC & 4593 & 40 & 5 & $0 \cdot 13$ & $0 \cdot 12$ & 4 \\
\hline NGC & 6891 & 40 & 5 & 0.13 & $0 \cdot 20$ & 5 \\
\hline IC & 4997 & 33 & 4 & $0 \cdot 11$ & $<0.09$ & 5 \\
\hline IC & 3568 & 32 & 5 & 0.11 & $<0.12$ & 5 \\
\hline NGC & 6894 & 32 & 5 & 0.11 & $0 \cdot 12$ & 8 \\
\hline $\mathbf{J}$ & 900 & 30 & 1 & $0 \cdot 10$ & $0 \cdot 10$ & 9 \\
\hline VV & 286 & 25 & 4 & 0.08 & - & - \\
\hline NGC & 6751 & 25 & 5 & 0.08 & $(-0.02)$ & 8 \\
\hline NGC & 7354 & 25 & 5 & 0.08 & 0.51 & 9 \\
\hline NGC & 2022 & 20 & 5 & 0.07 & 0.08 & 1 \\
\hline NGC & 6567 & 20 & 5 & 0.07 & $0 \cdot 14$ & 1 \\
\hline NGC & 6629 & 18 & 4 & 0.06 & $0 \cdot 17$ & 1 \\
\hline NGC & 1501 & 16 & 5 & 0.05 & $0 \cdot 19$ & 4 \\
\hline NGC & 6309 & 16 & 5 & 0.05 & $0 \cdot 18$ & 1 \\
\hline
\end{tabular}


Table 3 (continued)

$\begin{array}{cccc}\text { Pl. Neb. } & \begin{array}{c}\text { Corrected } \mathrm{H} \beta \text { Flux } \\ \left(10^{-12} \mathrm{erg} \mathrm{cm}^{-2} \mathrm{sec}^{-1}\right)\end{array} & \text { Reference } & \begin{array}{c}\text { Radio Flux Density } \\ \left(10^{-26} \mathrm{Wm}^{-2} \mathrm{~Hz}^{-1}\right)\end{array} \\ & \text { Predicted } & \text { Observed Reference }\end{array}$

$\begin{array}{lcccccc}\text { NGC } & 7008 & 16 & 5 & 0.05 & 0 \cdot 18 & 4 \\ \text { NGC } & 6537 & 14 & 1 & 0.05 ? & 0 \cdot 38 ? & 8 \\ \text { NGC } & 2371-2 & 13 & 5 & 0.04 & 0 \cdot 22 & 3 \\ \text { IC } & 5217 & 13 & 5 & 0.04 & 0 \cdot 15 & 5 \\ \text { BD } & 30^{\circ} 3639 & 13 & 5 & 0.04 & 0.44 & 4 \\ \text { NGC } & 6772 & 10 & 5 & 0.03 & 0.06 ? & 8 \\ \text { NGC } & 6563 & 1 *^{*} & 2 & >0.03 & 0 \cdot 13 & 5 \\ \text { NGC } & 6445 & 8 & 5 & 0.03 & 0 \cdot 27 & 5 \\ \text { J } & 320 & 8 & 5 & 0.03 & 0.08 ? & 8 \\ \text { NGC } & 6884 & 7 & 4 & 0.02 & 0.07 ? & 8 \\ \text { IC } & 351 & 6 & 5 & 0.02 & (-0.02) & 8 \\ \text { NGC } & 7139 & 6 & 5 & 0.02 & 0.07 ? & 8 \\ \text { NGC } & 6833 & 6 & 4 & 0.02 & 0.22 ? & 8 \\ \text { NGC } & 6058 & 5 & 5 & 0.02 & 0.01 & 8 \\ \text { VV } & 171 & 5 & 5 & 0.02 & - & - \\ \text { NGC } & 6072 & 4 * & 2 & >0.02 & 0.11 & 5 \\ \text { NGC } & 6886 & 3 & 4 & 0.01 & 0 \cdot 13 & 9 \\ \text { NGC } & 6807 & 3 & 4 & 0.01 & 0.05 & 1 \\ \text { NGC } & 6879 & 2 & 4 & 0.01 & 0.08 ? & 8 \\ \text { VV } & 267 & 2 & 4 & 0.01 & - & -\end{array}$

( ) Optically thick.

? Flux affected by confusion.

$(-)$ not detected.

* $\mathrm{H} \beta$ flux not corrected for reddening.

References for H $\beta$ fluxes: (1) Collins et al. (1961). (2) O’Dell (1963). (3) Osterbrock (1964). (4) Vorontsov-Velyaminov et al. (1964). (5) Seaton (1966).

References for Radio Data: (1) Slee and Orchiston (1965). (2) Khromov (1966). (3) Terzian (1966).

(4) Thompson et al. (1967). (5) Thompson and Colvin (1967). (6) Hughes (1967). (7) Terzian (1967).

(8) Davies et al. (1967). (9) Kaftan-Kassim (1967). (10) Ehman (1967).

Substituting numerical values in the last expression, and using $T_{\mathrm{e}}=10^{4}{ }^{\circ} \mathrm{K}, v=5000$ $\mathrm{MHz}$ to evaluate $\zeta$, assuming spherical symmetry for the observed nebula, and expressing $E$ in units of $\mathrm{cm}^{-6}$ parsec, $S_{v}$ in $\mathrm{Wm}^{-2} \mathrm{~Hz}^{-1}$, and $d$ (the diameter of the nebula) in degrees we have

$$
E=\frac{S_{v} T_{\mathrm{e}}^{1 / 2}}{2 \times 10^{-26} d^{2}}
$$

The emission measure of NGC 7027 is found to be $3.8 \times 10^{7} \mathrm{~cm}^{-6}$ parsec, that of NGC 6781 is $0 \cdot 11 \times 10^{5} \mathrm{~cm}^{-6}$ parsec. Thus, it is possible to derive estimates of densities and masses of planetary nebulae provided good distances and filling factors (fraction of nebula filled with matter) are available.

The detection of the hydrogen $109 \alpha$ recombination line $(5008.9 \mathrm{MHz})$ in $\mathrm{H}$ II regions by Höglund and Mezger (1965) has opened a new way of studying interstellar ionized 
hydrogen. Very recently Mezger et al. (1967) have succeeded in detecting the hydrogen $109 \alpha$ recombination line in the planetary nebula NGC 7027. From the ratio of line to continuum brightness temperatures an electron temperature of $11 \times 10^{3} \mathrm{~K}$ has been derived.

\section{Discussion}

The ratio of the emission coefficients at a radio frequency $v$ and at $\mathrm{H} \beta$ in a thermal region as shown by Terzian (1965) is

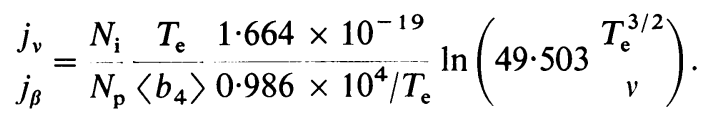

Since the planetary nebulae are optically thin at $\mathrm{H} \beta$ and at high radio-frequencies, the ratio $j_{v} \mid j_{\beta}$ is equal to the emergent fluxes at the corresponding wavelengths. The factor $\left\langle b_{4}\right\rangle$ indicates the degree of departure from thermodynamic equilibrium. The effective value of $\left\langle b_{4}\right\rangle$ for the $4 \rightarrow 2 \mathrm{H} \beta$ transition is given by Burgess (1958). We shall adopt a mean value of 0.20 for $\left\langle b_{4}\right\rangle$, which corresponds to Burgess' Case B (nebula optically thick in Lyman lines) at an electron temperature of $10^{4}{ }^{\circ} \mathrm{K}$. Using $5000 \mathrm{MHz}$ for $v$, a helium abundance of $15 \%$, and an electron temperature of $10^{4}{ }^{\circ} \mathrm{K}$, the ratio of the radio to $\mathrm{H} \beta$ emission is

$$
\begin{aligned}
& j_{v} \\
& j_{\beta}
\end{aligned}=3.28 \times 10^{-14} .
$$

The observed $\mathrm{H} \beta$ fluxes corrected for interstellar extinction taken from the literature were used to derive the radio fluxes of the bright planetary nebulae, using Equation (10). These are given in Table 3 together with the observed radio fluxes. It can easily be seen that there is good agreement between the observed and predicted flux densities. Table 3 includes 68 planetary nebulae with known $\mathrm{H} \beta$ fluxes. Only 9 of these have not been detected at radio frequencies. With fairly good accuracy the reported observed radio fluxes in Table 3 are the values at the optically thin part of the spectra of the planetary nebulae, except for NGC 6572, NGC 6905, and IC 418. These nebulae are still optically thick at the highest observed frequencies. In the case of NGC 6543, the radio observations at 5000,8000 and $16200 \mathrm{MHz}$ indicate that this nebula is optically thin and has a flux density of $0.9 \mathrm{f}$.u. The optical depth is unity at approximately $1500 \mathrm{MHz}$. The predicted radio flux for NGC 6543 from its $\mathrm{H} \beta$ flux is $1.57 \mathrm{f}$.u. This value seems high compared with the radio observations.

Since the ratio of the radio flux to that at $\mathrm{H} \beta$ is a function of the electron temperature we can get some estimates of the latter, by assuming the helium abundance in the nebulae. Figure 7 shows lines of constant electron temperatures in the radio and $\mathrm{H} \beta$ flux plane, where a helium abundance of $15 \%$ has been assumed. The observed optically thin radio fluxes and $\mathrm{H} \beta$ fluxes taken from Table 3 have been plotted in Figure 7 . Seventy-two percent of the nebulae show electron temperatures $>10^{4} \mathrm{~K}$, and almost 


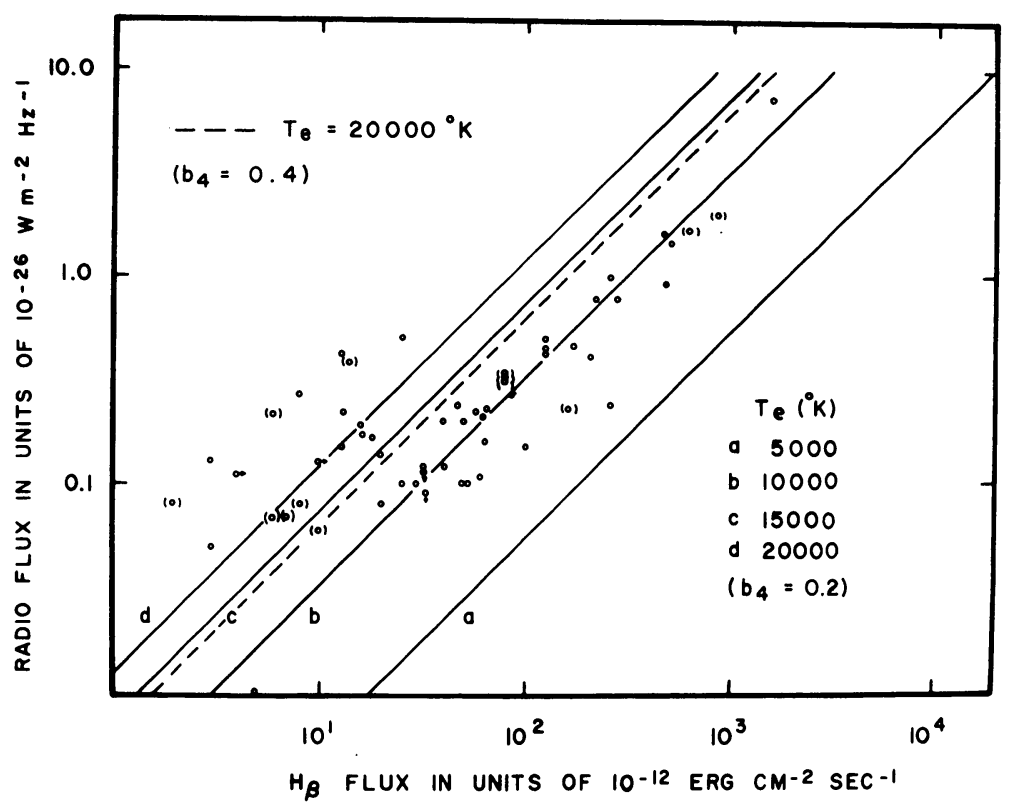

FIG. 7. A comparison between the radio and $H \beta$ fluxes. (The points in parenthesis have uncertain radio fluxes.)

all nebulae have electron temperatures $>7.5 \times 10^{3}{ }^{\circ} \mathrm{K}$. A few nebulae show temperatures above $2 \times 10^{4} \mathrm{~K}$; the radio-flux densities for some of these nebulae are uncertain - probably the observed radio fluxes are high due to confusion. However, NGC 7354, BD $30^{\circ} 3639$, NGC 2371-2, NGC 6445, NGC 6885, and NGC 6807 appear to have good radio-flux observations. One can also argue that the $\mathrm{H} \beta$ fluxes for these nebulae have been underestimated, particularly the estimated interstellar extinction corrections could be systematically low for these faint nebulae.

The constant-electron-temperature lines in Figure 7 were computed assuming $\left\langle b_{4}\right\rangle=0 \cdot 20$. This value is the best estimate for an electron temperature of $10^{4}{ }^{\circ} \mathrm{K}$. However, $\left\langle b_{4}\right\rangle$ increases with temperature and tends to make the electron temperature dependence on $j_{v} / j_{\beta}$ smaller. The dotted line in Figure 7 was computed using $\left\langle b_{4}\right\rangle=0.4$ for an electron temperature of $2 \times 10^{4}{ }^{\circ} \mathrm{K}$.

It should be pointed out that the lower limit of an accurate radio-flux measurement with present-day radio telescopes is of the order of $0 \cdot 1$ f.u. Figure 7 shows that most of the nebulae with very high electron temperatures were expected to have radio fluxes $\leqslant 0 \cdot 1 \mathrm{f}$.u. predicted from their $\mathrm{H} \beta$ fluxes. Certainly very accurate radio and optical measurements are needed for these nebulae in order to clarify the present results.

A few nebulae which have been observed to have relatively high radio fluxes, such 


\section{Table 4}

\section{A comparison of optically estimated extinction with the extinction derived from radio data}

\begin{tabular}{|c|c|c|}
\hline Pl. Neb. & $\begin{array}{c}\text { Measured } \\
\log F(\mathrm{H} \beta) \\
\left(\mathrm{erg} \mathrm{cm}^{-2} \mathrm{sec}^{-1}\right)\end{array}$ & $\begin{array}{r}\text { Optica } \\
\text { Extincti } \\
\Delta \log F(\mathrm{~F}\end{array}$ \\
\hline NGC 7027 & $-10 \cdot 12$ & 1.34 \\
\hline 418 & -9.53 & 0.4 \\
\hline NGC 6572 & -9.74 & 0.52 \\
\hline NGC 6853 & -9.44 & $0 \cdot 1$ \\
\hline NGC 6543 & -9.60 & $0 \cdot 2$ \\
\hline NGC 7293 & -9.35 & 0.0 \\
\hline NGC 7662 & -9.98 & 0.4 \\
\hline NGC 3242 & -9.81 & $0 \cdot 2$ \\
\hline NGC 7009 & -9.78 & $0 \cdot 1$ \\
\hline NGC 6826 & -9.92 & $0 \cdot 2$ \\
\hline NGC 6210 & -10.06 & 0.3 \\
\hline NGC 6804 & -11.28 & 0.9 \\
\hline NGC 6818 & $-10 \cdot 13$ & 0.4 \\
\hline IC 2149 & $-10 \cdot 50$ & 0.6 \\
\hline NGC 6720 & -10.06 & $0 \cdot 1$ \\
\hline IC $\quad 4634$ & -10.97 & 0.9 \\
\hline NGC 7026 & -10.90 & 0.8 \\
\hline NGC 6905 & -10.90 & 0.7 \\
\hline NGC 40 & $-10 \cdot 64$ & 0.4 \\
\hline NGC 6781 & $-11 \cdot 19$ & 1.0 \\
\hline NGC $650-1$ & -10.67 & 0.8 \\
\hline NGC 1535 & $-10 \cdot 36$ & $0 \cdot 2$ \\
\hline NGC 2392 & -10.39 & 0.4 \\
\hline NGC 6803 & $-11 \cdot 15$ & 0.92 \\
\hline IC 2165 & -10.99 & 0.7 \\
\hline NGC 3587 & $-10 \cdot 33$ & 0.0 \\
\hline NGC 4361 & $-10 \cdot 48$ & $0 \cdot 2$ \\
\hline NGC 6778 & $-11 \cdot 26$ & 0.9 \\
\hline NGC 6741 & -11.49 & $1 \cdot 15$ \\
\hline IC 4593 & -10.55 & 0.2 \\
\hline NGC 6891 & $-10 \cdot 60$ & $0 \cdot 6$ \\
\hline IC $\quad 4997$ & -10.49 & \\
\hline IC $\quad 3568$ & -10.82 & $0 \cdot 2$ \\
\hline NGC 6894 & -11.46 & 1.2 \\
\hline 900 & $-11 \cdot 27$ & 0.7 \\
\hline NGC 7354 & -11.55 & 0.7 \\
\hline NGC 2022 & $-11 \cdot 15$ & \\
\hline NGC 6567 & -10.93 & $1 \cdot 1$ \\
\hline NGC 6629 & -10.94 & \\
\hline NGC 1501 & $-11 \cdot 26$ & 1.8 \\
\hline NGC 6309 & $-11 \cdot 29$ & $0 \cdot 6$ \\
\hline NGC 7008 & $-10 \cdot 86$ & 1.0 \\
\hline NGC 6537 & $-11 \cdot 78$ & 0.9 \\
\hline NGC 2371-2 & -10.96 & 0.3 \\
\hline IC $\quad 5217$ & $-11 \cdot 18$ & 0.3 \\
\hline BD $\quad 30^{\circ} 3639$ & $-11 \cdot 50$ & 0.7 \\
\hline $\mathbf{N}$ & -11.65 & \\
\hline
\end{tabular}

$\begin{array}{cc}\text { Derived } \\ \text { Reference } & \text { Extinction } \\ \Delta \log F(\mathrm{H} \beta)\end{array}$

1.46

$>0.31$

$>0.45$

0.09

$>0.04$

0.04

0.36

0.28

0.16

0.03

0.21

$1 \cdot 13$ ?

0.28

0.68

0.17

0.63

0.82

0.89

0.66 ?

1.15

0.36

0.17

0.24

0.68

0.82

0.19

0.26

0.74

1.36

0.11

0.39

$(-0.07)$

$<0.38$

1.02

0.75

1.73

0.54

0.56

0.65

1.02

1.03

0.60

1.84 ?

0.79

0.84

1.63

0.91 ? 
Table 4 (continued)

$\begin{array}{lcccc}\text { Pl. Neb. } & \begin{array}{c}\text { Measured } \\ \log F(\mathrm{H} \beta) \\ \left(\mathrm{erg} \mathrm{cm}^{-2} \mathrm{sec}^{-1}\right)\end{array} & \begin{array}{c}\text { Optical } \\ \text { Extinction } \\ \Delta \log F(\mathrm{H} \beta)\end{array} & \text { Reference } & \begin{array}{c}\text { Derived } \\ \text { Extinction }\end{array} \\ \text { NGC 6563 } & -10.96 & - & 3 & \Delta \log F(\mathrm{H} \beta) \\ \text { NGC 6445 } & -11.20 & - & 3 & 0.56 \\ \text { J 320 } & -11.37 & 0.46 & 1 & 1.12 \\ \text { NGC 6884 } & -11.11 & 1.29 & 1 & 0.76 ? \\ \text { NGC 7139 } & -11.78 & 0.47 & 5 & 0.44 ? \\ \text { NGC 6833 } & -11.22 & - & 3 & 1.11 ? \\ \text { NGC 6058 } & -11.70 & 0.19 & 1 & 1.05 ? \\ \text { NGC 6072 } & -11.37 & - & 3 & 0.18 \\ \text { NGC 6886 } & -11.50 & 0.90 & 1 & 0.90 \\ \text { NGC 6879 } & -11.60 & 0.81 & 1 & 1.10\end{array}$

(1) Collins et al. (1961). (2) O’Dell (1962). (3) O’Dell (1963). (4) Osterbrock (1964). (5) Thompson et al. (1967).

as NGC 7635, NGC 6857, and IC 1470, are probably diffuse nebulae of small angular size and have not been included in Table 3. The planetary nebula NGC 6302 seems to be of particular interest since it possibly coincides with the X-ray source Sco XR-2 (Thompson and Colvin, 1967). No $\mathrm{H} \beta$-flux measurement is available for this nebula; however, the radio observations indicate that NGC 6302 is a thermal source.

For most of the brighter planetary nebulae, radio fluxes can be measured with an accuracy of $10 \%$ or better. From the radio measurements the $\mathrm{H} \beta$ fluxes can be predicted assuming the electron temperatures and helium composition. These then can be compared with the measured $\mathrm{H} \beta$ fluxes uncorrected for interstellar extinction, and the latter quantity can be derived. Table 4 shows a comparison of the optically estimated $\mathrm{H} \beta$ extinction and the extinction derived from the radio data. Figure 8 shows the comparison of the extinctions using only the reliable points from Table 4 . The correlation coefficient is $\mathbf{0 . 7 2}$. The solid straight line in Figure 8 is the expected correlation, and the dashed line is the best fit to the plotted points. The derived extinction $\Delta \log$ $F(\mathrm{H} \beta)$ for NGC 7354 , and $\mathrm{BD} 30^{\circ} 3639$ is more than twice as high as the extinction estimated optically. As was mentioned above, this can be the result of radio fluxes measured too high, or of underestimated $\mathrm{H} \beta$ fluxes. and (or) extinction corrections. In some cases where the helium abundances and electron temperatures can be found from optical observations accurate $\mathrm{H} \beta$ extinctions can be derived from the above method.

\section{Conclusion}

During the last few years planetary nebulae have been observed at radio frequencies. The results of these observations confirm the recombination theory of emission and have established planetary nebulae as thermal sources. 


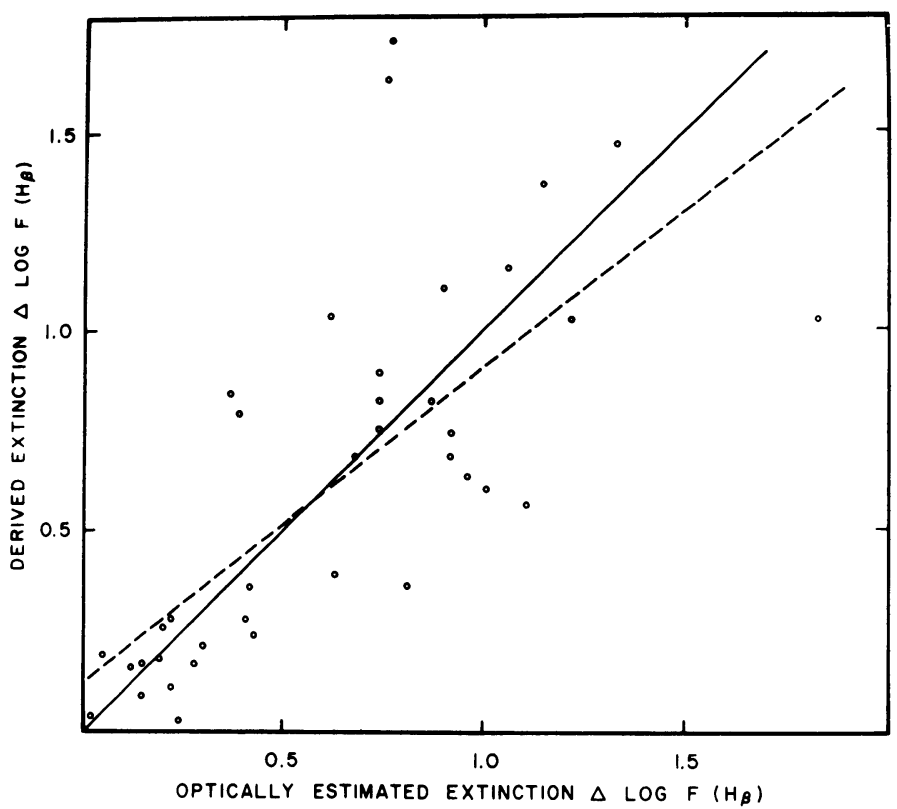

FIG. 8. A comparison between the optically estimated $H \beta$ interstellar extinction with the one derived from radio data.

More accurate observations are required in some cases, specially at the low radio frequencies in order to establish the optically thick part of the spectra of these nebulae.

Observations at the hydrogen $109 \alpha$ recombination line should be made, as well as at other hydrogen recombination lines. Some attempts should also be made to observe the $21-\mathrm{cm}$ line of neutral hydrogen, which may exist around some ionization-bounded planetary nebulae. The recently discovered strong $\mathrm{OH}$ emission in diffuse nebulae suggests that $\mathrm{OH}$ observations should be tried in planetary nebulae.

Finally it should be pointed out that observations to determine the brightnesstemperature distribution in planetary nebulae do not exist. Such observations require extremely high angular resolution, which present-day radio telescopes do not have. Presently there are two ways of observing the brightness-temperature distribution of a few planetary nebulae, one using the method of aperture synthesis, and the other using the method of lunar occultations. So far no lunar occultation of a planetary has been observed, but several will be occulted in the next few years.

\section{References}

Burgess, A. (1958) Mon. Not. R. astr. Soc., 118, 477.

Collins, G. W. III, Daub, C.T., O'Dell, C.R. (1961) Astrophys. J., 133, 471.

Davies, J.G., Ferriday, R.J., Haslam, C.G.T., Moran, M., Thomasson, P. (1967) Mon. Not. R. astr. Soc., 135, 139. 
Ehman, J.R. (1967) Astrophys. J. (in press).

Höglund, B., Mezger, P.G. (1965) Science, 150, 339.

Hughes, M.P. (1967) Astrophys. J., 149, 377.

Kaftan-Kassim, M.A. (1966) Astrophys. J., 145, 658.

Kaftan-Kassim, M.A. (1967) private communication.

Kellermann, I. K., Pauliny-Toth, I.I. K. (1967) private communication.

Khromov, G.S. (1966) Soviet Astr., 9, 705.

Lynds, C.R. (1961) Publ. nat. Radio Astr. Obs., 1, 85.

Menon, T.K., Terzian, Y. (1965) Astrophys. J., 141, 745.

Mezger, P.G., Altenhoff, W., Schraml, J., Burke, B.F., Reifenstein III, E.C., Wilson, T.L. (1967)

Astrophys. J., 150, L.157.

O'Dell, C.R. (1962) Astrophys. J., 135, 371.

O’Dell, C.R. (1963) Astrophys. J., 138, 293.

Oster, L. (1961) Astrophys. J., 134, 1010.

Osterbrock, D.E. (1964) A. Rev. Astr. Astrophys., 2, 95.

Seaton, M.J. (1966) Mon. Not. R. astr. Soc., 132, 113.

Slee, O. B., Orchiston, D. W. (1965) Austr. J. Phys., 18, 187.

Terzian, Y. (1965) Astrophys. J., 142, 135.

Terzian, Y. (1966) Astrophys. J., 144, 657.

Terzian, Y. (1967) Astr. J., 72, 443.

Thompson, A.R., Colvin, R.S. (1967) Astrophys. J., 150, 345.

Thompson, A. R., Colvin, R.S., Stanley, G.J. (1967) Astrophys. J., 148, 429.

Vorontsov-Velyaminov, B. A., Kostjakova, E. B., Dokuchaeva, O.D., Arhipova, V.P. (1964) Soviet Astr. 8, 196.

\section{DISCUSSION}

Minkowski: You mentioned NGC 40 as possibly non-thermal. Is there not a known non-thermal source, quite close by and visible on the Sky Survey plate, which is probably a supernova remnant?

Terzian: Yes, this is so, and it may be that the radio observations are confused.

Liller: Have there been any attempts to observe by their $21-\mathrm{cm}$ radiation the $\mathrm{H}_{\mathrm{I}}$ regions which must surround many planetaries?

Menon: Attempts to detect $21-\mathrm{cm}$ radiation associated with planetary nebulae have not been successful so far. Observationally it is a very difficult problem.

Thompson: Terzian has mentioned NGC 7635 as a case in which the present flux-density values show some deviation from a thermal spectrum. This nebula has recently been classified as diffuse by a number of people, and thus should not be included when considering evidence for the possible existence of non-thermal planetary nebulae.

Osterbrock: It must be remembered that the 'optical extinction' values quoted by Terzian are derived in some cases from photoelectrically measured Balmer-line or Balmer/Paschen-line ratios, while in other cases they are simply estimates based on galactic latitude. 